\title{
VIEWPOINT \\ Health Disparities and the Coronavirus Disease 2019 (COVID-19) Pandemic in the USA
}

\section{Sameed Ahmed M. Khatana, MD, MPH ${ }^{1,2}$ and Peter W. Groeneveld, MD, MS $S^{2,3}$}

'Division of Cardiovascular Medicine, Perelman School of Medicine, University of Pennsylvania Philadelphia, PA, USA; ${ }^{2}$ The Leonard Davis Institute of Health Economics, University of PennsylvaniaPhiladelphia, PA, USA; ${ }^{3}$ Division of General Internal Medicine, Perelman School of Medicine, University of PennsylvaniaPhiladelphia, PA, USA.

$\mathrm{J}$ Gen Intern Med 35(8):2431-2

DOI: $10.1007 / \mathrm{s} 11606-020-05916-\mathrm{w}$

(c) Society of General Internal Medicine 2020

$\mathrm{T}$ he coronavirus disease 2019 (COVID-19) pandemic has quickly demonstrated the many shortcomings of the US healthcare system. Based on evidence from the H1N1 influenza pandemic of 2009, it will likely exacerbate many of the disparities in healthcare access and outcomes that exist in the USA. Unlike every other developed country in the world, a large number of people in the USA still lack health insurance (27.5 million people as of 2018), a disproportionate number of whom are people of lower socioeconomic status and racial and ethnic minorities. Additionally, over half of non-elderly adults in the USA receive employer-sponsored health insurance, making them vulnerable to a loss of healthcare access in the event of losing their employment. Minority and lower socioeconomic status populations also experience a high burden of chronic cardiovascular and pulmonary disease that will place them at a higher risk of complications from infection. Urgent actions, such as expansion of health insurance coverage, need to be taken to lessen the burden of this pandemic and the accompanying economic consequences on vulnerable populations.

The US experience with the H1N1 influenza pandemic demonstrated racial and ethnic minorities were at a higher risk of infection, partly because they were more likely to live in dense urban areas. ${ }^{1}$ Uninsured persons with H1N1 were also more likely to delay access to care and to use emergency departments, rather than seek care through an established primary care provider. ${ }^{2}$ For COVID-19, this raises the potential of uninsured patients increasing the risk of infection among already stretched emergency department staff. Delays in seeking care may also complicate efforts in tracking the spread of the infection and potentially increase exposure to others around them. During the H1N1 pandemic, patients from minority and lower socioeconomic status populations

Received April 10, 2020

Accepted May 6, 2020

Published online May 27, 2020 were also more likely to be hospitalized, in part related to their higher burden of underlying chronic diseases. ${ }^{3}$ Patients without health insurance were also less likely to receive antiviral therapy and have lower vaccination rates, ${ }^{4,5}$ which may become critical to public health when these potentially become available for COVID-19. Although COVID-19 is a unique pandemic with different patterns of transmission and outcomes than previous viral respiratory pandemics, many of the unfortunate patterns seen previously are likely to repeat themselves. There is already some evidence that black Americans are disproportionately being impacted by the COVID-19 pandemic. In Illinois, $28.4 \%$ of confirmed cases and $42.9 \%$ of deaths are black, despite representing $14 \%$ of the state population. ${ }^{6}$ Similarly, in Louisiana, around 70\% of COVID-19related deaths have been among black residents despite comprising only $32 \%$ of the state population. ${ }^{7}$

Beyond the direct impact of infection, the current pandemic is likely to have major economic consequences. Unemployment statistics released by the Bureau of Labor Statistics indicate that in March 2020, the USA experienced its largest month-to-month increase in unemployment since January 1975. During the most recent economic downturn in the USA - the Great Recession of 2008-2009—-black and Hispanic individuals experienced significantly higher unemployment rates than white individuals, suggesting that this likely is to happen again. This not only will result in direct health impacts from loss of household income but also will increase the number of uninsured people in the USA due to the loss of employer-sponsored health insurance.

Federal and state policy makers have taken some steps to improve healthcare access, particularly for low-income individuals. This has included making COVID-19 testing free through the Families First Coronavirus Response Act and granting waivers to individual states to streamline enrollment into Medicaid (the primary source of government funded health insurance for low-income individuals in the USA). However, perhaps the most significant mechanism to increase access to health insurance for lowincome and vulnerable populations is the expansion of Medicaid eligibility as allowed for under the Patient Protection and Affordable Care Act (ACA). Expansion of Medicaid eligibility under the ACA resulted in 15 million low-income individuals gaining health insurance 
coverage. This expansion has been associated in improvements in various health measures including mortality rates in certain populations. However, 14 states have still not expanded Medicaid eligibility. These states, predominantly in the US South and Midwest, are home to an estimated 2.8 million individuals who may gain health insurance coverage if eligibility were to be expanded. Many of these states also have large minority populations, as well as a high proportion of their residents with pre-existing chronic conditions, which is likely to translate into a relatively greater benefit of insurance expansion in these states. Although one of the concerns around expansion of Medicaid has been its impact on state budgets, the ACA allows for the federal government to provide $100 \%$ of the funding for additional enrollees (which decreases to $90 \%$ over the subsequent years). Additionally, hospitals in Medicaid non-expansion states provide a significantly greater amount of uncompensated care. With growing concern about the impact of the COVID-19 on the financial health of certain hospitals, particularly safety net hospitals that provide a disproportionate amount of care to vulnerable populations, due to the decrease in elective procedures, Medicaid expansion will also provide an economic lifeline to such hospitals.

Patients in the US healthcare system experience disparities in access, care, and outcomes in several areas. The US experience with the H1N1 pandemic demonstrated that this is no different for respiratory viral pandemics. As the current COVID-19 pandemic progresses, it will disproportionately impact not only the health of vulnerable individuals but also their economic wellbeing. Actions taken now by policy makers, such as expansion of health insurance for low-income individuals, can have a significant impact in impacting health disparities, thereby enhancing public health benefits for vulnerable populations.

Corresponding Author: Sameed Ahmed M. Khatana, MD, MPH; Division of Cardiovascular Medicine, Perelman School of Medicine, University of Pennsylvania Philadelphia, PA, USA (e-mail: sameed. khatana@pennmedicine.upenn.edu).

\section{Compliance with Ethical Standards:}

Conflict of Interest: Dr. Khatana's work is supported by funding from the National Heart, Lung, and Blood Institute (5T32HL007843). Dr. Groeneveld has no disclosures.

\section{REFERENCES}

1. Quinn SC, Kumar S, Freimuth VS, Musa D, Casteneda-Angarita N, Kidwell K. Racial disparities in exposure, susceptibility, and access to health care in the US H1N1 influenza pandemic. Am J Public Health. 2011;101(2):285-93; doi:https://doi.org/10.2105/AJPH.2009.188029

2. Jehn M, Kim Y, Bradley B, Lant T. Community knowledge, risk perception, and preparedness for the 2009 influenza A/H1N1 pandemic. J Public Health Manag Pract. 2011;17(5):431-8; doi:https://doi.org/10. 1097/PHH.Ob013e3182113921

3. Thompson DL, Jungk J, Hancock E, Smelser C, Landen M, Nichols M, et al. Risk factors for 2009 pandemic influenza A (H1N1)-related hospitalization and death among racial/ethnic groups in New Mexico. Am J Public Health. 2011;101(9):1776-84; doi:https://doi.org/10.2105/AJPH.2011. 300223

4. Franklin JM, Choudhry NK, Uscher-Pines L, Brill G, Matlin OS, Fischer MA, et al. Equity in the receipt of oseltamivir in the United States during the H1N1 pandemic. Am J Public Health. 2014;104(6):1052-8; doi:https://doi.org/10.2105/AJPH.2013.301762

5. Takayama M, Wetmore CM, Mokdad AH. Characteristics associated with the uptake of influenza vaccination among adults in the United States. Prev Med. 2012;54(5):358-62; doi:https://doi.org/10.1016/j.ypmed.2012. 03.008

6. Illinois Department of Health. COVID-19 statistics. https://www.dph. illinois. gov/covid19/covid19-statistics. Accessed April 7, 2020

7. Louisiana Department of Health - Office of Public Health. Louisiana coronavirus (COVID-19) information. http://ldh.la.gov/Coronavirus/. Accessed April 7, 2020

Publisher's Note Springer Nature remains neutral with regard to jurisdictional claims in published maps and institutional affiliations. 